\title{
Impact of cold dissection tonsillectomy method combined with selective low voltage bipolar diathermy cauterization on the post tonsillectomy pain in the pediatric age group
}

\author{
Ramesh Varadharajan*, Vaishnav Radhakrishnan, Sneha Suresh, Sreedevi S. Babu
}

Department of Otolaryngology, Head and Neck Surgery, Aarupadai Veedu Medical College and Hospital, Kirumampakkam, Puduchery, India

Received: 27 August 2019

Revised: 06 September 2019

Accepted: 16 September 2019

\section{*Correspondence:}

Dr. Ramesh Varadharajan,

E-mail: rameshent@gmail.com

Copyright: () the author(s), publisher and licensee Medip Academy. This is an open-access article distributed under the terms of the Creative Commons Attribution Non-Commercial License, which permits unrestricted non-commercial use, distribution, and reproduction in any medium, provided the original work is properly cited.

\begin{abstract}
Background: Pediatric tonsillectomies are commonly performed by cold dissection or electro cautery dissection. Both the techniques have been compared in many articles for their role in controlling the post-operative pain and bleeding. With the electro cautery dissection the blood loss is comparatively less, but the post-operative pain is reported as more. Several expensive new techniques are available to address the problems. A simple technique of cold dissection tonsillectomy combined with selective low voltage bipolar diathermy cauterization performed in the pediatric age group is being studied retrospectively for its impact in managing the post-operative pain.

Methods: The case records of 73 patients aged 4-15 years who were operated for tonsillectomy from January 2018 to July 2019 are retrospectively studied. 33 patients underwent cold dissection and ligature tonsillectomy and 40 patients underwent tonsillectomy by cold dissection combined with selective low voltage bipolar diathermy cauterization. The postoperative pain incidence in these two groups is studied.

Results: The cold dissection tonsillectomy combined with selective low voltage bipolar diathermy cauterization technique was found to be useful in managing the postoperative pain in pediatric tonsillectomies.

Conclusions: Performing cold dissection tonsillectomy with precise mucosal incision, gentle dissection and combining it with selective low voltage bipolar diathermy cauterization of the bleeding points was found to be helpful in controlling the postoperative pain in pediatric tonsillectomies. Prescribing a syrup form of NSAID from the second post-operative day was found to be of additional help.
\end{abstract}

Keywords: Tonsillectomy, Cold dissection, Bipolar diathermy, Post tonsillectomy pain

\section{INTRODUCTION}

Tonsillectomy was performed as early as in 1000 B.C. ${ }^{1}$ In $20^{\text {th }}$ century dissection tonsillectomy was first described by Worthington and Waugh. ${ }^{2,3}$ Ligatures to control bleeding were introduced by Cohen in $1909 .{ }^{4}$ Bipolar diathermy tonsillectomy was introduced by Pang in 1995. ${ }^{5}$ Newer surgical techniques using radiofrequency or laser have evolved over time and are being practiced by many.
Cold dissection tonsillectomy with ligatures for bleeding is still being practiced widely for its low cost and less postoperative pain. The ligature technique needs expertise and could be time consuming. The incidence of reactionary hemorrhage after tonsillectomy was observed to increase three folds after the use of ligatures to control bleeding by Carmody et al. ${ }^{6}$ Tonsillectomy by electro cautery dissection reduces the intra operative blood loss but increases the postoperative pain as observed by 
several authors in their studies and recently by Moffatteh et al in 2018 .

The advantages of less postoperative pain observed with cold dissection and less intra operative bleeding observed with diathermy cauterization are incorporated into this simple combined technique.

The objective of this study was to study retrospectively the impact of a simpler surgical technique of combining a classical cold dissection tonsillectomy with a selective bipolar diathermy cauterization in the management of post tonsillectomy pain in Pediatric age group.

\section{METHODS}

The case records of 73 patients aged 4-15 years who underwent Tonsillectomy between January 2018 to July 2019 at the Department of Otolaryngology Head \& Neck Surgery, Aarupadai Veedu Medical College Hospital, Puduchery were reviewed. All patients had been randomly selected and operated by two different surgeons. All patients had undergone tonsillectomy by cold dissection and snare technique. In 33 patients silk ligatures were used to control the intra operative bleeding (method A). In the remaining 40 patients selective bipolar diathermy with 20 watts power setting was used to control the bleeding (method B).

Case records of children between the age group of 4-15 and who were operated for various symptoms like snoring, recurrent throat pain and otitis media were included in this study.

Case records of children above the age of 15 and adults who were operated with a past history of peritonsillitis or abscess formation and those who were operated for other conditions such as Eagle's syndrome and those with suspected malignancy were excluded from this study as the severity and duration of post tonsillectomy pain will be of a greater magnitude in such cases.

General anesthesia with naso tracheal intubation was used. Rose tonsillectomy position and Boyle Davis mouth gag was used. Adenoidectomy was performed if indicated by the curettage method using a St. Clair Thompson's adenoid curettes and the bleeding was controlled by gauze packs.

In method A (ligature tonsillectomy) the upper pole of the tonsil was grasped with a tonsil holding forceps and pulled medially. Using a toothed dissecting forceps the initial mucosal incision was precisely made $1 \mathrm{~mm}$ medial to the anterior pillar, in the loose areolar tissue between the tonsil capsule and the anterior pillar avoiding injury to the fibers of the palatoglossus muscle. The tonsil capsule was identified and the upper pole gently enucleated using a Mollison's enucleator. Further blunt dissection was carried out towards the lower pole of the tonsil using the blunt end of Mollison's enucleator. The dissection was kept close to the tonsil to avoid damage to the muscle fibers in the tonsillar bed. Tonsil fossa was packed with cotton balls to control bleeding. The tonsil was removed using a tonsil snare. The bleeding points were caught with a tonsil artery forceps and ligated using 3-0 silk. Similar procedure was repeated on the other side also.

In method B (diathermy tonsillectomy) after the initial mucosal incision the upper pole of the tonsil was enucleated and dissection carried out in the same way as in method A. During dissection any bleeding points found were quickly cauterized for 2-3 seconds with a bipolar diathermy forceps with a power setting of 20 watts and packed with cotton balls. The cautery point was carefully applied close to the tonsil. The bleeding points found in the tonsillar bed were also cauterized in the same way. To minimize thermal injury to the muscles of the tonsillar bed, cautery was selectively used only to cauterize the bleeding points and not the entire fossa. After controlling the bleeding the fossa was packed with cotton balls and similar procedure repeated on the other side.

After completion of the surgery the adenoid packs and the tonsil fossa packs were removed and nasal cavity was suctioned with a rubber catheter to remove clots. The mouth gag was released half way and tonsil fossae were inspected for any fresh bleeding points, which were controlled by ligature or cauterization as per the method followed. Before extubation the tonsil fossae were again inspected for any bleeding points or clots and were managed appropriately.

All the patients were shifted to the recovery suite and observed for 1 hour and transferred to the respective wards. Pulse and other vital signs were monitored on a half hourly basis for the first 4 hours and then on a hourly basis for the next 4 hours. Intravenous antibiotic (coamoxiclav or ceftriaxone) was prescribed according to the weight. Intravenous fluids in the form of 5\% dextrose or dextrose saline was given according to the weight for the first 24 hours after surgery.

Cold oral fluids were introduced 8 hours after surgery and the patient's response to the act of swallowing in terms of crying with pain, vomiting and refusal to take the oral fluids are noted and recorded. The duty Nurses were instructed to give one dose of diclofenac sodium Inj. or Suppositories according to the weight and recorded the frequency of further doses administered. Vomiting was managed with inj. ranitidine and inj. ondansetron administered as per the weight.

On the first post-operative day, the severity of the early morning throat pain during oral fluids was monitored and one dose of diclofenac sodium inj. or suppositories were given according to the weight and recorded. The nurses were instructed to record the number additional analgesics requested for pain. Intravenous antibiotic was 
continued and syrup Ibuprofen with paracetamol every 8 hours was prescribed according to the weight. Vital signs and urine output were monitored routinely.

On the second post-operative day, the morning throat pain during fluid soft diet intake was monitored and the nurses were instructed to give analgesic injections only on a P.R.N. basis and record the number analgesics given. Intravenous antibiotics and oral Ibuprofen with paracetamol syrup were continued. Soft diet and oral fluids were encouraged and the vital signs and urine output were monitored routinely.

All the patients were discharged on the third postoperative day with appropriate dietary advice, proper postoperative instructions and a prescription of syrup coamoxiclav or cefixime and syrup ibuprofen with paracetamol according to the weight for 3 days. They were reviewed after 3 days in the E.N.T. outpatient department. The symptoms of throat pain, fever, and food intake were noted. The patients were reviewed again after 7 days and advised to resume normal activities.

Statistical analysis was done with SISA (http://www.quantitativeskills.com/sisa/) and the mean analgesic doses prescribed for pain control is calculated. ${ }^{8}$

\section{RESULTS}

There were a total of 73 patients who underwent surgery and there were 39 male $(53 \%)$ and 34 female (47\%) patients. The gender wise distribution of total patients is shown in Table 1 . There were $4(5.5 \%)$ patients in the $0-5$ year age group and $39(53.4 \%)$ patients in the 6-10 year age group and $30(41.1 \%)$ patients in the $11-15$ year age group.

Table 1: Gender distribution of total 73 patients.

\begin{tabular}{|ll|}
\hline Sex & Numbers $(\%)$ \\
\hline Males & $39(53)$ \\
\hline Females & $34(47)$ \\
\hline Total & $73(100)$ \\
\hline
\end{tabular}

Method A tonsillectomy was done in a total of 33 patients. There was $1(3 \%)$ patient in the $0-5$ age group, $10(30.30 \%)$ patients in the 6-10 age group and 22 $(66.70 \%)$ patients in the $11-15$ years age group.

Method B tonsillectomy was done in a total of 40 patients. There were $3(7.5 \%)$ patients in the $0-5$ age group, $29(72.5 \%)$ patients in the 6-10 age group and 8 $(20 \%)$ patients in the $11-15$ years age group.

The total number of analgesic doses prescribed for the 73 patients in the first 24 hours after surgery was 162 . Statistical analysis showed the mean analgesic dose prescribed in the first 24 hours after surgery as per Table 2.

The total number of analgesic doses prescribed to the 33 patients operated by method A was 72 and the mean value was 2.18 .

The total number of analgesic doses prescribed to the 40 patients operated by method B was 90 and the mean value was 2.25 .

Table 2: The mean number of analgesic doses prescribed in the postoperative period.

\begin{tabular}{|c|c|c|c|c|c|c|c|c|c|c|c|}
\hline \multicolumn{12}{|c|}{ Means table } \\
\hline Method & Mean & SD & Variance & \multicolumn{2}{|c|}{ Std. err } & \multicolumn{3}{|c|}{$95 \%$ z-CI. } & Freq. & $\%$ & $++\%$ \\
\hline A & 2.181818 & 0.391675 & 0.153409 & \multicolumn{2}{|c|}{0.068182} & \multicolumn{2}{|c|}{2.048184} & 2.315452 & 33 & 45.21 & 45.21 \\
\hline B & 2.25 & 0.438529 & 0.192308 & \multicolumn{2}{|c|}{0.069338} & & & 2.385899 & 40 & 54.79 & 100 \\
\hline All & 2.219178 & 0.416552 & 0.173516 & \multicolumn{2}{|c|}{0.048754} & & & 2.314734 & 73 & 100 & 100 \\
\hline \multicolumn{12}{|c|}{ Skewness/Kurtosis table } \\
\hline \multirow{2}{*}{ Method } & \multirow{2}{*}{ Mean } & \multicolumn{2}{|c|}{ Skewness } & \multicolumn{5}{|c|}{ Kurtosis } & & & \\
\hline & & \multicolumn{2}{|c|}{ Sample } & Population & \multicolumn{2}{|c|}{ Std. err } & \multicolumn{2}{|c|}{ Sample } & \multicolumn{2}{|c|}{ Population } & Std. err \\
\hline A & 2.181818 & 1.65 & \multicolumn{2}{|c|}{1.73} & \multicolumn{2}{|c|}{0.4086} & \multicolumn{2}{|c|}{0.722} & 1.05137 & \multicolumn{2}{|c|}{0.7984} \\
\hline $\mathrm{B}$ & 2.25 & 1.155 & \multicolumn{2}{|c|}{1.2} & \multicolumn{2}{|c|}{0.3738} & \multicolumn{2}{|c|}{-0.667} & -0.59175 & \multicolumn{2}{|c|}{0.7326} \\
\hline All & 2.219178 & 1.358 & \multicolumn{2}{|c|}{1.386} & 0.2 & & \multicolumn{2}{|c|}{-0.157} & -0.08117 & \multicolumn{2}{|c|}{0.5552} \\
\hline
\end{tabular}

Table 3: Age wise distribution of additional dose of postoperative analgesics prescribed in the first postoperative day to patients operated by method $A$ and $B$.

\begin{tabular}{|lll|}
\hline Age (years) & Method A & Method B \\
\hline $\mathbf{0 - 5}$ & $\mathbf{N}(\boldsymbol{\%})$ & $\mathbf{N}(\boldsymbol{\%})$ \\
\hline $\mathbf{6 - 1 0}$ & $0(0)$ & $0(0)$ \\
\hline $\mathbf{1 1 - 1 5}$ & $0(0)$ & $2(6.90)$ \\
\hline Total additional doses & $6(27.27)$ & $8(100)$ \\
\hline
\end{tabular}


None of the patients operated by both methods required any additional injectable analgesics or suppositories from the second post-operative day, other than the oral nonsteroidal anti-inflammatory drug (NSAID) syrup prescribed. They were able to take oral fluid and soft diet and maintain adequate urine output. There were no incidences of reactionary or secondary hemorrhage and all the patients were discharged on the third postoperative day.

\section{DISCUSSION}

The majority of the tonsillectomies are performed in the pediatric age group for various problems. Pediatric tonsillectomy in rural practice has some special considerations. Children from rural areas often present with a poor nutritional status and low body weight. Postoperative throat pain is one of the common problems and children often refuse food and fluids due to the pain and in hot climates could get dehydrated. ${ }^{9}$ Hence adequate pain control is essential. Children's responses to pain are different from adults as shown in the study by Tay. ${ }^{10}$ Self-reported pain scores collection in children in the immediate postoperative period is difficult and young children cannot use the scales effectively as reported by Maunuksela et al. ${ }^{11}$ Trained nurses are invaluable in assessing the need for analgesic medications in the postoperative wards especially in the pediatric age group.

Tonsillectomies are commonly performed by the cold dissection technique and ligatures are still used to control bleeding. It has the advantage of less postoperative pain but the operating time and the blood loss during and after surgery are reported to be more in several studies. The technique of applying ligatures needs skill and difficult in children due to the smaller size of the oral cavity and limited space. It could be time consuming and the incidence of reactionary hemorrhage after tonsillectomy was observed to increase three folds after the use of ligatures to control bleeding by Carmody et al. ${ }^{6}$ In this study intra operative use of low voltage bipolar diathermy during dissection helped to overcome this problem.

Several electro dissection tonsillectomy techniques have evolved over time and various methods like monopolar or bipolar diathermy, cobalation, harmonic scalpel and laser are used widely. Though the newer tonsillectomy techniques like radiofrequency, laser or harmonic scalpel are reported to produce less postoperative pain, their use in rural practice becomes restricted due to the higher cost of the equipment.

Diathermy dissection tonsillectomy is less expensive but has been shown to be associated with more post-operative pain in several studies. The post-operative pain after electro cautery dissection tonsillectomy using monopolar or bipolar cautery could be due to the higher power setting, duration and frequency of the cautery application as explained by Walker et al. ${ }^{12}$ The use of electro dissection needle or the forceps for the entire dissection could result in more thermal injury and tissue damage, delaying the healing and increased pain after surgery. This was shown in the studies by Dyleski et al, Paradise and Raut et al. ${ }^{13-15}$

This problem has been addressed by the selective use of the bipolar diathermy forceps during tonsillectomy (method B). It has been used, only to cauterize the bleeding points during surgery and not for dissection. The duration of the cautery application also has been restricted to a brief period of 2-3 seconds. The diathermy voltage has been specifically maintained at a lower level with a power setting of 20 watts. These points were meticulously observed with a scope of minimizing the thermal injury. This probably resulted in lesser tissue damage and less post-operative pain.

Sharif et al in their comparative study have concluded that tonsillectomy using bipolar diathermy is an effective method with no measurable increase in the post-operative morbidity when compared to the dissection snare method. ${ }^{16}$ Their findings are confirmed in this study by the minimal difference in the mean values of postoperative analgesic prescriptions used in the first 24 hours after surgery (group A 2.18 and group B 2.25).

Injury to the palatoglossal muscle fibers located in the anterior pillar due to an improper incision and injury to the muscles in the tonsillar bed due to improper dissection are some of the other reasons suggested for the severity of post tonsillectomy pain. To avoid such injuries, several techniques like careful mucosal incision avoiding the palatoglossal muscle fibers, maintaining the blunt dissection close to the tonsil and careful handling of the tonsillar bed during cauterization have been meticulously followed during surgery in this study.

The post tonsillectomy pain following electro dissection has been reported to be less on the second and third postoperative days. Ali et al in their study have observed that the pain increases later on the $7^{\text {th }}$ and $14^{\text {th }}$ day after surgery. ${ }^{17}$ This phenomenon could be due to deeper thermal injury and deeper tissue inflammations. But in our study all the patients were comfortable on the $7^{\text {th }}$ day and $14^{\text {th }}$ day reviews, taking a normal diet with minimal pain and have resumed normal life.

Alkhalil et al in their study in 2018 have concluded that the problem of postoperative pain after electro dissection tonsillectomy could be managed by prescribing a NSAID from the second post-operative day. ${ }^{18}$ Similarly in this study oral NSAID (syrup ibuprofen + paracetamol) has been prescribed from the second post-operative day for better pain management.

Most of the patients could be managed with the oral NSAID alone from the second postoperative day without the need for any additional analgesic injections or suppositories. During the first review after discharge from the hospital, which was a week after surgery the patients were comfortable and taking a normal diet with minimal complaints of pain. 
In rural areas, due to the problems of patient compliance with respect to the dosage and duration of antibiotics, the previous throat infections could have been partially cleared, creating a chronic low grade residual infection. The post-operative pain was shown to be increased in patients operated with a history of recurrent tonsillitis in the study by Alkhalil et al. ${ }^{18}$ This finding was also observed in this study wherein the children above the age of 10 have been prescribed more number of additional analgesic injections for post-operative pain irrespective of the method of surgery.

The presence of a low grade residual infections and inflammations would explain the need for additional analgesic requirement in older children. To cover such residual infections intravenous antibiotics have been prescribed in the pre-operative and post-operative period in this study.

Tonsillectomy is being practiced as a day care surgery in many centers. In rural areas factors like level of literacy, remote location of the patient's home, availability of a quick transport facility and accessibility to the medical facilities in case of an emergency are to be considered. Keeping those factors in mind the patients in this study have been kept in the hospital till the third postoperative day to provide a proper postoperative antibiotic cover to prevent secondary infections and discharged and reviewed at fixed intervals.

\section{CONCLUSION}

Cold dissection tonsillectomy using conventional instruments are simple to perform and cost effective. Bipolar cautery is a less expensive addition and an effective tool in any surgeons' armamentarium. The problem of bleeding and post tonsillectomy pain in Pediatric cases can be effectively managed by using the bipolar cautery for 2-3 seconds in a low voltage setting to cauterize the bleeding points during surgery and to observe a careful selective cauterization of the tonsillar bed during a cold dissection tonsillectomy. We find it a less expensive but an effective technique. Prescription of NSAID syrup from the second postoperative day is additionally helpful in the pain management.

\section{ACKNOWLEDGEMENTS}

We wish to acknowledge and thank the Management, Dean and Medical Superintendent of our institution for the permission and the Professor and Staff of the ENT department, the Medical Records Officer and his staff for their kind co-operation in conducting this study.

Funding: No funding sources Conflict of interest: None declared Ethical approval: Not required

\section{REFERENCES}

1. Young J, Bennett J. History of tonsillectomy. ENT News. 2004;13:34-5.

2. Worthington TC. A simple method of excision of the faucial tonsil. JAMA. 1907;48:1761-2.

3. Waugh G. A simple operation for the complete removal of tonsils, with notes on 900 cases. Lancet. 1909;173:1314-5.

4. Cohen L. Postoperative tonsillar bleeding. Its surgical control, with mention of cases. JAMA. 1909;53:696-8.

5. Pang Y. Pediatric tonsillectomy: bipolar electro dissection and dissection/ snare compared. J Laryngol Otol. 1995;109:733-6.

6. Carmody D, Vamadevan T, Cooper SM. Post tonsillectomy hemorrhage. J Laryngol Otol. 1982;96:635-8.

7. Moffatteh MR, Salehi F, Hosseini M, Taheri MH, Sharifzadeh G, Taheri MH. Comparison of postoperative morbidity between conventional cold dissection and bipolar electrocautery tonsillectomy: which technique is better? Brazilian J Otorhinolaryngol. 2019;753:1-7

8. SISA. Available at: http://www.quantitativeskills. com/sisa/. Accessed on 3 June 2019.

9. Thung AK, Elmaraghy CA, Barry N, Tumin D, Jatana KR, Rice J, et al. Double blind randomized placebo controlled trial of single dose intravenous Acetaminophen for pain associated with Adeno tonsillectomy in Pediatric patients with sleep Disordered-breathing. J Pediatr Pharmacol Therap. 207;22(5):344-51.

10. Tay HL. Post-operative morbidity in electro dissection tonsillectomy. J Laryngol Otol. 1195;109:209-11.

11. Maunuksela E-L, Olkkola KT, Korpela R. Measurement of pain in children with self reporting and behavioral assessment. Clin Pharmacol Ther. 1987;42:137-41.

12. Walker P, Gillies D. Post Tonsillectomy hemorrhage rates: Are they technique dependent? Otolaryngol Head Neck Surg. 2007;136:S27-S31.

13. Dyleski R, Dennis M. Tonsillitis, tonsillectomy and adenoidectomy. Atlas of Head and Neck Surgery Otolaryngology. 2nd edition. Lippincott Williams and Wilkins. 2001: 979-993.

14. Paradise JL. Tonsillectomy and adenoidectomy. Pediatr Otolaryngol. 1996;2:1054-65.

15. Raut V, Bhat N, Kinsella J, Toner JG, Sinnathuray AR, Stevenson M, et al. Bipolar scissors versus cold dissection tonsillectomy: A Prospective, Randomized, Multi Unit Study. The Laryngoscope. 2001;111(12):2178-82.

16. Sharif M, Zaman J, Yousaf N, Iqbal K. Diathermy tonsillectomy versus conventional dissection tonsillectomy. J Post Graduate Med Institute. 2001;15(1):84-90.

17. Ali M, Rafique A, Dastgir M, Rashid M, Maqbool S, Bashir S. Comparison of bipolar electrocautery 
and cold steel dissection methods for tonsillectomy. PAFM J. 2014;64:34-8.

18. Alkhalil ARY, Mohammad SM, Allela OQB. Post Tonsillectomy Pain of Bipolar Electrocautery and Cold steel Dissection:A Randomized Prospective Comparative Study. J Pharm Pract Community Med. 2018;4(2):121-3.
Cite this article as: Varadharajan $\mathrm{R}$, Radhakrishnan V, Suresh S, Babu SS. Impact of cold dissection tonsillectomy method combined with selective low voltage bipolar diathermy cauterization on the post tonsillectomy pain in the pediatric age group. Int $\mathbf{J}$ Otorhinolaryngol Head Neck Surg 2019;5:1470-5. 